\title{
Nonsaturating extreme magnetoresistance and large electronic magnetostriction in LuAs
}

\author{
J. Juraszek $\odot,{ }^{1}$ L. Bochenek $\odot,{ }^{1}$ A. Rudenko $\odot,{ }^{1}$ M. M. Hosen, ${ }^{2}$ M. Daszkiewicz $\odot,{ }^{1}$ Z. Wang,,${ }^{3,}$ J. Wosnitza, ${ }^{3}$ Z. Henkie, ${ }^{1}$ \\ M. Samsel-Czekała $\odot,{ }^{1}$ M. Neupane, ${ }^{2}$ and T. Cichorek $\oplus^{1}$ \\ ${ }^{1}$ Institute of Low Temperature and Structure Research, Polish Academy of Sciences, 50-422 Wroctaw, Poland \\ ${ }^{2}$ Department of Physics, University of Central Florida, Orlando, Florida 32816, USA \\ ${ }^{3}$ Hochfeld-Magnetlabor Dresden (HLD-EMFL) and Würzburg-Dresden Cluster of Excellence ct.qmat, \\ Helmholtz-Zentrum Dresden-Rossendorf, 01328 Dresden, Germany
}

(Received 2 August 2019; published 7 November 2019)

\begin{abstract}
In the known topological semimetals, conventional charge carriers exist in addition to relativistic quasiparticles, and thus a disentangling of their conduction properties remains challenging. Here, we address an unsaturated extreme magnetoresistance (XMR) with a marked deviation from the semiclassical $B^{2}$ behavior that is commonly credited to the presence of topologically protected electronic states. For the topologically trivial semimetal LuAs, we observe a nonsaturating XMR with a nonquadratic magnetic-field dependence gained up to nearly $60 \mathrm{~T}$. Remarkably, this diamagnetic material exhibits a very large magnetostriction that provides solid evidence for a field-dependent variation of electron and hole concentrations. We show that an underlying strain-induced change in the charge-carrier densities can give rise to an unsaturated XMR even in a moderately imbalanced semimetal. Our finding is of importance as well for topological semimetals in which the number of conventional charge carriers can be continuously altered with increasing field, and hence some of their high-field properties may not necessarily reflect the presence of massless quasiparticles.
\end{abstract}

DOI: 10.1103/PhysRevResearch.1.032016

The last decade has experienced considerable effort in the synthesis of materials with unconventional band structures, initiated by studies of graphene and topological insulators. Most recently, great interest is directed toward other exotic and topologically protected phases - Dirac and Weyl semimetals. In topological quantum semimetals linearly dispersing conduction and valence bands intersect at points or along lines in momentum space, and hence are supposed to allow the propagation of electron waves that behave as Dirac and Weyl fermions in the bulk. Many Dirac and Weyl semimetals show exceptional properties, such as an electrical conductivity higher than noble metals, high charge-carrier mobilities and extreme magnetoresistance (XMR) with field dependences varying between quasilinear [1-4] and near quadratic [5-11]. However, important contributions to their transport phenomena may come from numerous trivial charge carriers that exist as well in topological semimetals. An interesting but not easily accessible way to unmask relativistic quasiparticles is to drive a topological semimetal far into its quantum limit where these quasiparticles may be studied in isolation $[12,13]$. Much less extreme conditions are needed for studying closely related but topologically trivial semimetals. This way also offers a wider spectrum of experimental probes with high

\footnotetext{
*Present address: High Magnetic Field Laboratory, Chinese Academy of Sciences, Hefei, Anhui 230031, China.

Published by the American Physical Society under the terms of the Creative Commons Attribution 4.0 International license. Further distribution of this work must maintain attribution to the author(s) and the published article's title, journal citation, and DOI.
}

accuracy for in-depth understanding of background properties driven from a small overlap of parabolic bands.

The rediscovery of XMR in nonmagnetic monopnictides has drawn attention to these binary compounds, with Fermi surfaces (FSs) [14-16] much simpler than those of elemental arsenic, antimony, and bismuth, as ideal model systems to investigate the field-induced properties of semimetals. $\mathrm{LaBi}$ and $\mathrm{LaSb}$ as well as YSb exhibit nonsaturating magnetoresistances as large as the extreme MRs in topological semimetals [17-19], although the measured band structure appears to be inverted only in LaBi [20-24]. Moreover, their field dependences of the magnetoresistance are substantially different from a semiclassical two-band $B^{2}$ behavior [25-28]. So far, different mechanisms, such as a considerable imbalance between electron and hole concentrations [9,27] and large differences in charge-carrier mobilities [26], have been proposed to explain the subquadratic behavior. However, as shown for LaSb with $\mathrm{XMR} \propto B^{1.78}$ [25], only a multiband scenario with a high degree of fine tuning [29] appears to account for an unsaturated field dependence also in extremely large magnetic fields. But it requires a perfect electron-hole compensation that is inconsistent with the experimentally observed diversity of amplitudes and field dependences [17-28,30].

In this Rapid Communication, we report on a study of the magnetic-field-dependent transport and thermodynamic properties of the diamagnetic semimetal LuAs [31]. Single crystals of LuAs display an XMR $\propto B^{1.65}$ of $400000 \%$ without any sign of saturation in magnetic fields up to $58.5 \mathrm{~T}$ (at $5 \mathrm{~K}$ ). Angle-resolved photoemission spectroscopy (ARPES) and first-principles calculations consistently establish that topologically nontrivial electronic states are absent in LuAs. In addition to this, we observe a very large magnetostriction 

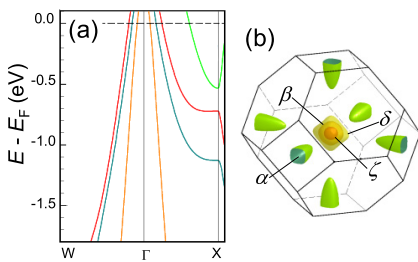
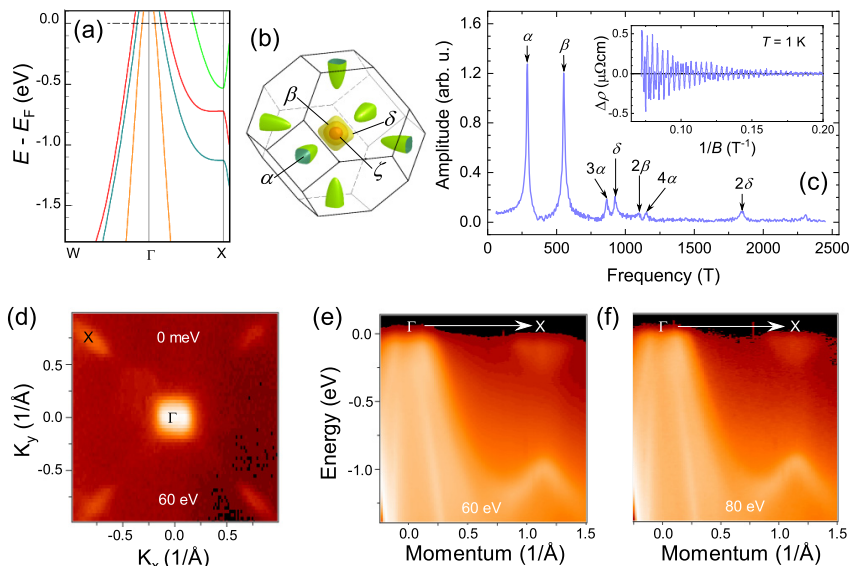

FIG. 1. Fermi surface and band structure of LuAs. (a) Calculated band structure along the $W-\Gamma-X$ line. (b) Fermi surface in the first Brillouin zone composed of three hole pockets $(\beta, \delta, \zeta)$ and threefold degenerate electron ellipsoids $(\alpha)$. (c) FFT spectrum of SdH oscillations showing three principal frequencies $F_{\alpha}=285 \mathrm{~T}, F_{\beta}=548 \mathrm{~T}$, $F_{\delta}=930 \mathrm{~T}$, and their harmonics. Inset: Corresponding oscillatory part of the electrical resistivity measured at $1 \mathrm{~K}$ for $j \|[100]$ and $B \|$ [001]. (d) Fermi-surface mapping in the $K_{x}-K_{y}$ plane measured by use of ARPES. (e), (f) Dispersion maps along the $\Gamma-X$ direction manifesting a large energy gap around the $\Gamma$ point.

$\left(\sim 10^{-5}\right.$ at $\left.10 \mathrm{~T}\right)$ that provides thermodynamic evidence for a field-induced change in carrier density. We show that underlying strain-dependent effects can account for the unsaturated MR even in the case of a moderately imbalanced semimetal.

In zero field, LuAs exhibits a metallic temperature dependence of the electrical resistivity $\rho(T)$ with a residual resistivity as low as $\rho_{0}=520(50) \mathrm{n} \Omega \mathrm{cm}$ and a large residual resistivity ratio $\mathrm{RRR}=\rho_{300 \mathrm{~K}} / \rho_{0} \simeq 73$ (sample $1-1$ ) manifesting the high quality of our crystals [cf. Fig. 2(a)]. The high electrical conductivity together with the small Sommerfeld coefficient of the electronic specific heat $\gamma=$ $0.38(2) \mathrm{mJ} \mathrm{K}^{-2} \mathrm{~mol}^{-1}$ and a strong diamagnetic signal of the low-temperature magnetization indicate that LuAs is a nonmagnetic semimetal [see Supplemental Material (SM) [35] for further details on the basic properties]. The semimetallic character of LuAs is further corroborated by the electronic structure near the Fermi level $E_{\mathrm{F}}$. Figure 1(a) presents the band-structure calculated fully relativisically along the $W-\Gamma-X$ line. Similar to other rare-earth monopnictides $[18,19,23,24]$, there are several bands crossing $E_{\mathrm{F}}$ : three hole bands in the vicinity of the $\Gamma$ point and one electron band around the $X$ point. However, in striking contrast to related monopnictides revealing XMR, the conduction band and the valence bands do not cross between $\Gamma$ and $X$ points, but are well separated by a direct gap of about $0.2 \mathrm{eV}$ at the $X$ point. As shown in Fig. 1(b), the Fermi surface of LuAs consists of four sheets in the first Brillouin zone (BZ). Three of them, centered at the $\Gamma$ point (labeled $\beta, \delta, \zeta$ ), are holelike and originate predominantly from the As $4 p$ states, while the electronlike pocket (labeled $\alpha$ ) is composed of three equivalent ellipsoids around the $X$ point driven mainly from the Lu $5 d$ states. A conservative estimation of the chargecarrier concentrations yields about $2 \times 10^{20} \mathrm{~cm}^{-3}$. The

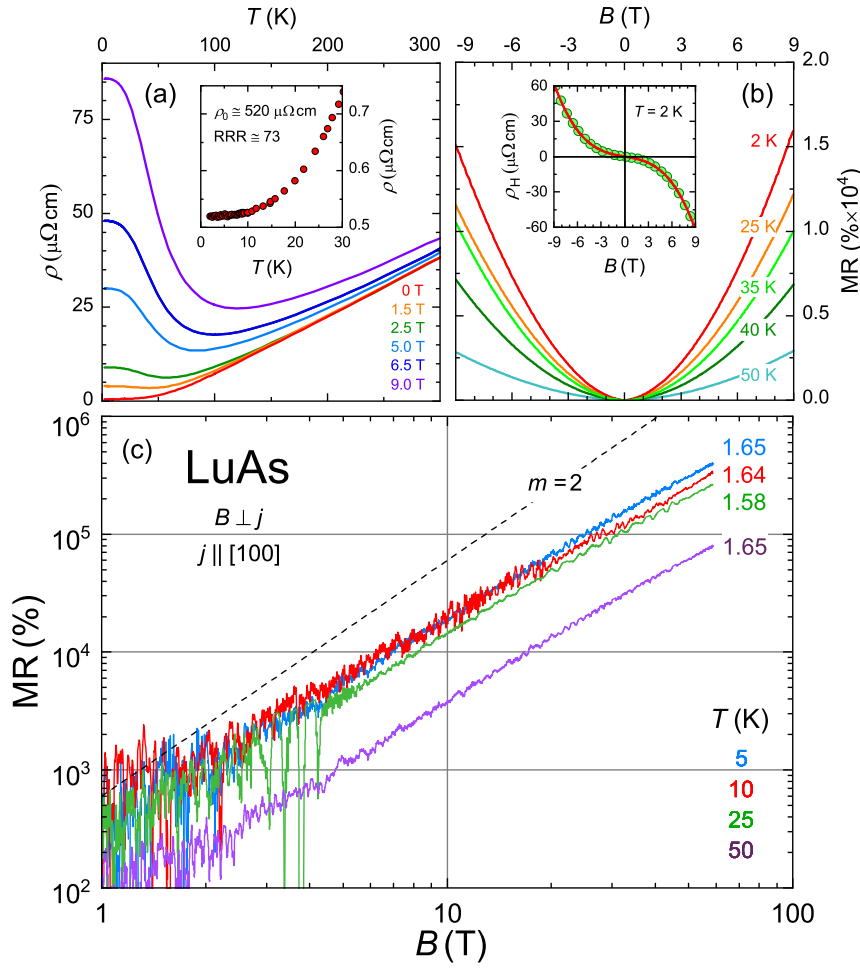

FIG. 2. Magnetoresistance of LuAs. (All data were obtained for sample 1-1.) (a) Temperature dependence of the electrical resistivity $\rho$ at different transverse magnetic fields. Inset: Enlargement of the low- $T$ zero-field $\rho$ data. (b) Relative change of the $B$-dependent resistivity normalized to the zero-field value at several temperatures below $50 \mathrm{~K}$. (No off-diagonal components were subtracted.) Inset: Field-dependent Hall resistivity $\rho_{\mathrm{H}}$ at $2 \mathrm{~K}$. The solid red line is a fit to the two-band model. (c) Extremely large, nonsaturating magnetoresistance of LuAs up to $58.5 \mathrm{~T}$ at several temperatures. Note the substantial deviation of the transverse MR from the semiclassical $B^{2}$ behavior.

calculated frequencies correspond with the fast Fourier transform (FFT) spectrum obtained from the Shubnikov-de Haas ( $\mathrm{SdH})$ oscillations [cf. Fig. 1(c)], except for the principal frequency $F_{\zeta} \approx 272 \mathrm{~T}$ for the smallest hole pocket that has not been detected in our experiments (see the SM for a more detailed discussion).

In addition, we performed ARPES measurements which evidence that LuAs lacks topological protection. Figure 1(d) shows the FS intensity plot which reveals the presence of multiple pockets near $E_{\mathrm{F}}$. Specifically, we observed a rectangular Fermi-surface pocket at the BZ center and elliptical pockets along the $X-\Gamma-X$ direction. Figures 1(e) and 1(f) show the dispersion maps along the $\Gamma-X$ direction recorded with photon energies 60 and $80 \mathrm{eV}$, respectively. While our experimental observations confirm the $a b$ initio prediction of three holelike bands crossing the Fermi level, a minor but significant difference concerns the size of the direct energy gap at the $X$ point that has been measured to be as large as $0.7 \mathrm{eV}$. This unambiguously rules out the presence of topologically protected electronic states in LuAs. We also remark that the measured energy gap along the $\Gamma-X$ direction in LuAs is by a factor of nearly 2 larger than in LaAs with 
magnetoresistance orders of magnitude smaller as compared to LaSb and LaBi $[23,36]$.

As shown in Fig. 2(a), in a magnetic field perpendicular to the applied current, the temperature dependence of the electrical resistivity is completely altered. Upon cooling, the resistivity strongly increases and exhibits a plateau below about $20 \mathrm{~K}$. The resistivity rise becomes more and more pronounced as the field increases, which leads to a large magnetoresistance $\mathrm{MR}=[\rho(B)-\rho(0)] / \rho(0)$ at low temperatures. Figure 2(b) depicts the MR measured in transverse magnetic fields up to $9 \mathrm{~T}$ at various temperatures. At $T=2 \mathrm{~K}$, we find that LuAs exhibits a magnetoresistance as large as $16000 \%$. (An even larger $M R \simeq 24000 \%$ was found for the sample 2-2; see Fig. S6.) With increasing temperature, the transverse MR decreases dramatically to just about $16 \%$ at $300 \mathrm{~K}$ and $9 \mathrm{~T}$ (not shown). One of the most remarkable results for LuAs is presented in Fig. 2(c). The transverse MR is still increasing at $58.5 \mathrm{~T}$, where the MR is $400000 \%$ (at $5 \mathrm{~K}$ ), reflecting the extraordinary character of LuAs [37]. However, in contrast to the quadratic field dependence expected for a nontopological semimetal, the magnetoresistance of LuAs (sample 1-1) grows as $B^{m}$ with $m=1.65$ up to the highest fields measured. We note that similar observations were made for all other samples regardless of the field direction and the crystal quality, though some differences are found in the amplitude of MR and the exponent $m$ that, in a few cases, varies with temperature and can be as small as 1.41 (see Supplemental Material [35] for more MR results).

Nonsaturating XMRs that significantly diverge from the quadratic form have been reported for different Weyl semimetal candidates as well as certain nonmagnetic monopnictides, but most experiments were limited to relatively moderate fields of 9 or $14 \mathrm{~T}$, where an unsaturated behavior is easily achieved, even if the hole $n_{h}$ and electron $n_{e}$ densities differ by several percent [1-3,8-10,17-19,25-28]. Indeed, a nonsaturating XMR requires a so-called resonance at $n_{h} \simeq n_{e}$ and this stringency becomes more and more severe with increasing field, as has been pointed out earlier [7]. This was illustrated, e.g., for YSb for which XMR $\propto B^{1.85}$ was ascribed in a semiclassical two-band framework as the cooperative action of a very large electron-hole imbalance $\left(n_{h} / n_{e}=0.81\right)$ and an immense difference between hole and electron mobilities $\left(\mu_{h} / \mu_{e} \approx 250\right)$, without giving any evidence for the assumed mobility difference [26]. Moreover, this simple scenario fails above around $7 \mathrm{~T}$ where a deviation from the perfect electron-hole compensation necessarily implies an obvious trend to saturation [26]. For similar reasons, an exact compensation must be fulfilled for a more complex three-band model that was proposed to explain XMR $\propto B^{1.78}$ of LaSb in fields between 3 and $40 \mathrm{~T}$, although details of the mobility difference were not provided [25]. We emphasize, however, that a multiband model does not result in generally new physics, i.e., its low- and high-field forms are expected to follow a two-band quadratic MR [29]. In this context, the very recent MR results $\left(\simeq 3 \times 10^{8} \%\right.$ at $56 \mathrm{~T}$ and $\left.2 \mathrm{~K}\right)$ for antimony should be noted [38]. For this indeed near-perfectly compensated semimetal $\left(n_{h} / n_{e} \simeq 0.999\right)$ with very anisotropic FS, the field dependence of the nonsaturating MR is close to quadratic ( $m \simeq 1.95$ ) and even such a slight difference from a semiclassical framework requires a substantial field dependence of the mobility tensors [38].

A moderate (i.e., few percent) electron-hole imbalance is suggested for LuAs by the sample-independent observation of an unsaturated XMR, regardless of the level of disorder and details of the $B^{m}$ behavior. A violation of the perfect compensation is further supported by the observation of a large unsaturated MR $\propto B^{1.65}$ at $T \leqslant 50 \mathrm{~K}$ where the chargecarrier densities strongly vary with temperature, as evidenced from Hall-effect measurements (see Supplemental Material [35]). Besides for a narrow temperature range (75-120 K), the Hall voltage is negative (at $9 \mathrm{~T}$ ) and, more importantly, the field-dependent Hall resistivity $\rho_{\mathrm{H}}(B)$ is strongly nonlinear. This is shown in the inset of Fig. 2(b) where the $\rho_{\mathrm{H}}(B)$ data at $2 \mathrm{~K}$ are well described by a two-band model assuming the moderate imbalance ratio $n_{h} / n_{e}=0.96$. We imposed the fit (red line) to capture both experimental findings $\rho_{0} \approx 520 \mathrm{n} \Omega \mathrm{cm}$ and $\mathrm{MR} \approx 20000 \%$ at $9 \mathrm{~T}$. From this fit $(R$-square $=0.99994)$ we obtain $n_{e}=2.70 \times$ $10^{20} \mathrm{~cm}^{-3}, \mu_{e}=3.14 \times 10^{4} \mathrm{~cm}^{2} \mathrm{~V}^{-1} \mathrm{~s}^{-1}$, and $\mu_{h}=1.33 \times$ $10^{4} \mathrm{~cm}^{2} \mathrm{~V}^{-1} \mathrm{~s}^{-1}$. While these carrier densities are similar to $n_{h}$ and $n_{e}$ of related monopnictides [17-19,24,26,28,30], $\mu_{e}$ and $\mu_{h}$ differ only by a factor of $\sim 2.4$.

Taking advantage of the recent progress in high-resolution capacitance dilatometry [39], we measured the field-induced length change $\Delta L / L$ of LuAs. In diamagnetic materials the magnetostriction is due to the charge carriers and, in contrast to transport properties, this thermodynamic probe directly couples to the density of states at $E_{\mathrm{F}}$. If all charge carriers belong to a single band, the electronic magnetostriction is very small, though still detectable $\left(\sim 10^{-9}\right.$ at $\left.10 \mathrm{~T}\right)$. However, for a multiband material with a multivalley structure such as graphite and pure $\mathrm{Bi}$ [40-42], this thermodynamic probe is greatly enhanced $\left(\sim 7 \times 10^{-6}\right.$ at $\left.10 \mathrm{~T}\right)$ by a field-dependent band overlap and a resulting variation of electron and hole concentrations. The relation between the magnetostriction and the field-induced change in charge-carrier density $\Delta n(B)$ takes the simple form

$$
\Delta L / L=c \Delta n(B)
$$

where the magnitude of the material-dependent coefficient $c$ is determined by a product of components of elastic compliance and deformation potential tensors for a given orientation of an applied field with respect to the crystallographic axes.

As shown in Fig. 3(a), the field-induced expansions in LuAs are extraordinarily large though this material is far below the quantum limit. Indeed, $\Delta L / L$ in LuAs measured along the [100] axis at $25 \mathrm{mK}$ is positive, amounts to about $1 \times 10^{-5}$ at $B=10 \mathrm{~T}$, and is essentially unaltered at $4.2 \mathrm{~K}$ (not shown). The expansion measured for $B \perp$ [100] is by a factor of 2 smaller than the longitudinal magnetostriction, indicating that the sample volume does not differ substantially for different field orientations. However, both sets of data display different $B$ dependences. For $B \| \Delta L$, the field dependence is quadratic. For $B \perp \Delta L$, the magnetostriction shows a $B^{1.75}$ behavior without any sign of saturation up to $16 \mathrm{~T}$. As discussed below, the differences seen in the parallel and perpendicular configurations reflect an anisotropy of field-induced edge shifts of the three electron valleys located around the $X$ point of the BZ. 


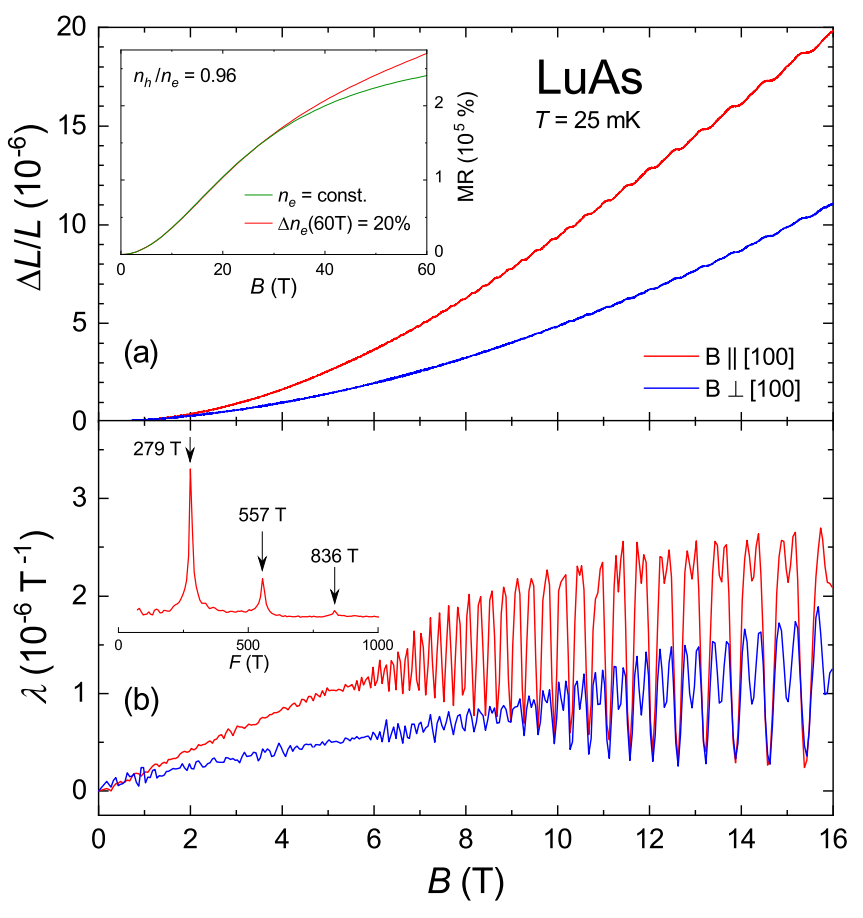

FIG. 3. Magnetostriction in the diamagnetic semimetal LuAs. (a) Magnetic-field dependence of the relative length change $\Delta L / L$ measured along the [100] direction at $25 \mathrm{mK}$ both in the parallel and perpendicular configuration. Inset: Expected effect of a field-dependent increase of the electron and hole concentrations on the magnetoresistance of a moderately imbalanced semimetal. (b) Magnetostriction coefficient $\lambda$ measured for $B \| \Delta L$ (red line) and $B \perp \Delta L$ (blue line). Inset: FFT amplitudes of the oscillatory magnetostriction along the [100] axis observed at $25 \mathrm{mK}$.

Following the outline developed in Ref. [40], we roughly estimate the net changes of electron $\Delta n_{e}$ and hole $\Delta n_{h}$ densities in LuAs due to the transverse field (the charge neutrality requires $\Delta n_{e}=\Delta n_{h}$ ). Using Eq. (1) and assuming that elasticcompliance and deformation-potential tensors result in a coefficient $c \approx 1.5 \times 10^{-24} \mathrm{~cm}^{3}$ similar to those reported for graphite and $n$-type germanium [40,42], we obtain $\Delta n_{e} / n_{e} \approx$ $\Delta n_{h} / n_{h} \approx 1 \%$ at $B=10 \mathrm{~T}$. Such changes in charge-carrier densities with respect to their zero-field values appear to be too small to make any visible contributions to other properties, in particular, to the magnetoresistance. However, if one assumes that the $B$ dependences of the magnetostriction are not significantly altered in larger fields, then one could expect $\Delta n_{e}=\Delta n_{h}$ of $\sim 23 \%$ at $60 \mathrm{~T}$. Such changes in the electron and hole concentrations are large enough to play an important role in the XMR.

To illustrate this point, we performed numerical simulations using a two-band model with the densities and the mobilities obtained from the Hall-effect analysis. The results are displayed in the inset of Fig. 3(a). As expected for $n_{h} / n_{e}=$ 0.96 , the MR shows an obvious trend to saturation above around $30 \mathrm{~T}$ (green line). However, this high-field behavior is dramatically altered when field-induced changes in the charge-carrier densities are considered. The red curve signifies the total MR obtained assuming a $B^{1.75}$ increase of $n_{h}$ and $n_{e}$ by 1 and $20 \%$ at 10 and $60 \mathrm{~T}$, respectively. As a result, we observe a magnetoresistance that does not show a tendency to saturation.

By contrast to other properties, quantum oscillations in the magnetostriction are caused only by strain-dependent extremal cross-section areas of the Fermi surface [43]. For LuAs, quantum oscillations are clearly visible already in the raw $\Delta L / L$ data [cf. Fig. 3(a)] and even more sharply discernible in the derived coefficient $\lambda=\frac{1}{L} \frac{d L}{d B}$ [cf. Fig. 3(b)], where different amplitudes and different local maxima/minima ratios found in the parallel and perpendicular configurations additionally underline the anisotropy of fieldinduced strains. FFT analysis, performed on the oscillatory part of the longitudinal data, reveals only one fundamental frequency $F_{\alpha}=279 \mathrm{~T}$ and its two harmonics $F_{2 \alpha}=557 \mathrm{~T}$ and $F_{3 \alpha}=836 \mathrm{~T}$ that correspond to the minimal extremal cross section of the electron ellipsoids [cf. inset to Fig. 3(b)]. The absence of other frequencies in the oscillatory magnetostriction indicates that the electron pockets are strongly pressure dependent, while the hole pockets hardly change with pressure. Since this effect does reduce the complexity of the oscillatory spectrum, the third harmonic can be resolved with reasonable accuracy. Thus, using a Dingle-temperatureindependent variant of the harmonic ratio method $[44,45]$, we estimate an effective $g$-factor of about 6 for the electrons in LuAs when $B \|[100]$. This value is considerably larger than the free-electron value $g \simeq 2$ and this enhancement is in line with the field-induced edge shifts of the electron valleys as a driving mechanism for the charge-density change.

Though not yet fully understood, the relationship between the large magnetostrictive strains and the lack of saturation of the XMR in the imbalanced semimetal LuAs implies a shift of the Fermi level. Therefore, one can suppose that field-induced effects associated with the strain dependence of conventional charge carriers would result in even more interesting observations in topological semimetals. This seems particularly true for low-carrier-density semimetals such as $\mathrm{TaP}, \mathrm{NbP}$, and $\mathrm{ZrSiS}$ with Weyl or Dirac points located only several meV away from $E_{\mathrm{F}}[4,11,46]$.

In conclusion, we have presented a comprehensive study on the diamagnetic semimetal LuAs with a trivial electronic band structure, and yet displaying an unsaturated and subquadratic XMR up to nearly $60 \mathrm{~T}$. Equally remarkable is a very large magnetostriction which provides thermodynamic evidence for a field-induced change of carrier densities. We showed that underlying strain-dependent processes can play an important role in the high-field transport properties as well, giving rise to a nonsaturating XMR even in a moderately imbalanced semimetal. Thus, our finding shows that a change of the Fermi energy due to the magnetostrictive strain should be considered when a Weyl semimetal is exposed to high magnetic fields.

We would like to thank Thomas Lorenz and Tomasz Durakiewicz for helpful discussions. This work was supported by the Polish National Science Centre (Project No. 2016/21/B/ST3/02361). We also acknowledge support by HLD at HZDR, member of the European Magnetic Field Laboratory (EMFL). J.W. is supported by the DFG through the Würzburg-Dresden Cluster of Excellence on Complexity and Topology in Quantum Matter - ct.qmat (EXC 2147, Project ID 
No. 39085490). M.N. is supported by the Air Force Office of Scientific Research under Award No. FA9550-17-1-0415 and the National Science Foundation (NSF) CAREER Award No. DMR-1847962.
[1] T. Liang, Q. Gibson, M. N. Ali, M. Liu, R. J. Cava, and N. P. Ong, Nat. Mater. 14, 280 (2015).

[2] X. Huang, L. Zhao, Y. Long, P. Wang, D. Chen, Z. Yang, H. Liang, M. Xue, H. Weng, Z. Fang, X. Dai, and G. Chen, Phys. Rev. X 5, 031023 (2015).

[3] S. S.-L. Zhang, G. Vignale, and S. Zhang, Phys. Rev. B 92, 024412 (2015).

[4] C. Shekhar, A. K. Nayak, Y. Sun, M. Schmidt, M. Nicklas, I. Leermakers, U. Zeitler, Y. Skourski, J. Wosnitza, Z. Liu, Y. Chen, W. Schnelle, H. Borrmann, Y. Grin, C. Felser, and B. Yan, Nat. Phys. 11, 645 (2015).

[5] X. Wang, X. Pan, M. Gao, J. Yu, J. Jiang, J. Zhang, H. Zuo, M. Zhang, Z. Wei, W. Niu, Z. Xia, X. Wan, Y. Chen, F. Song, Y. Xu, B. Wang, G. Wang, and R. Zhang, Adv. Electron. Mater. 2, 1600228 (2016).

[6] N. Kumar, Y. Sun, N. Xu, K. Manna, M. Yao, V. Süss, I. Leermakers, O. Young, T. Förster, M. Schmidt, H. Borrmann, B. Yan, U. Zeitler, M. Shi, C. Felser, and C. Shekhar, Nat. Commun. 8, 1642 (2017).

[7] M. N. Ali, J. Xiong, S. Flynn, J. Tao, Q. D. Gibson, L. M. Schoop, T. Liang, N. Haldolaarachchige, M. Hirschberger, N. P. Ong, and R. J. Cava, Nature (London) 514, 205 (2014).

[8] N. J. Ghimire, Y. Luo, M. Neupane, D. J. Williams, E. D. Bauer, and F. Ronning, J. Phys.: Condens. Matter 27, 152201 (2015).

[9] E. Mun, H. Ko, G. J. Miller, G. D. Samolyuk, S. L. Bud'ko, and P. C. Canfield, Phys. Rev. B 85, 035135 (2012).

[10] K. Wang, D. Graf, L. Li, L. Wang, and C. Petrovic, Sci. Rep. 4, 7328 (2014)

[11] F. Arnold, C. Shekhar, S. C. Wu, Y. Sun, R. D. dos Reis, N. Kumar, M. Naumann, M. O. Ajeesh, M. Schmidt, A. G. Grushin et al., Nat. Commun. 7, 11615 (2016).

[12] B. J. Ramshaw, K. A. Modic, A. Shekhter, Y. Zhang, E.-A. Kim, P. J. W. Moll, M. Bachmann, M. K. Chan, J. B. Betts, F. Balakirev, A. Migliori, N. J. Ghimire, E. D. Bauer, F. Ronning, and R. D. McDonald, Nat. Commun. 9, 2217 (2018).

[13] C.-L. Zhang, C. M. Wang, Z. Yuan, X. Xu, G. Wang, C.-C. Lee, L. Pi, C. Xi, H. Lin, N. Harrison, H.-Z. Lu, J. Zhang, and S. Jia, Nat. Commun. 10, 1028 (2019).

[14] H. Kitazawa, T. Suzuki, M. Sera, I. Oguro, A. Yanase, A. Hasegawa, and T. Kasuya, J. Magn. Magn. Mater. 31, 421 (1983).

[15] A. Hasegawa, J. Phys. Soc. Jpn. 54, 677 (1985)

[16] T. Kasuya, M. Sera, and T. Suzuki, J. Phys. Soc. Jpn. 62, 2561 (1993).

[17] F. F. Tafti, Q. D. Gibson, S. K. Kushwaha, N. Haldolaarachchige, and R. J. Cava, Nat. Phys. 12, 272 (2016).

[18] F. F. Tafti, Q. Gibson, S. Kushwaha, J. W. Krizan, N. Haldolaarachchige, and R. J. Cava, Proc. Natl. Acad. Sci. USA 113, E3475 (2016).

[19] N. J. Ghimire, A. S. Botana, D. Phelan, H. Zheng, and J. F. Mitchell, J. Phys.: Condens. Matter 28, 235601 (2016).
[20] N. Kumar, C. Shekhar, S.-C. Wu, I. Leermakers, O. Young, U. Zeitler, B. Yan, and C. Felser, Phys. Rev. B 93, 241106(R) (2016).

[21] X. H. Niu, D. F. Xu, Y. H. Bai, Q. Song, X. P. Shen, B. P. Xie, Z. Sun, Y. B. Huang, D. C. Peets, and D. L. Feng, Phys. Rev. B 94, 165163 (2016)

[22] J. Nayak, S.-C. Wu, N. Kumar, C. Shekhar, S. Singh, J. Fink, E. E. D. Rienks, G. H. Fecher, S. S. P. Parkin, B. Yan, and C. Felser, Nat. Commun. 8, 13942 (2017).

[23] T. J. Nummy, J. A. Waugh, S. P. Parham, Q. Liu, H.-Y. Yang, H. Li, X. Zhou, N. C. Plumb, F. F. Tafti, and D. S. Dessau, npj Quantum Mater. 3, 24 (2018).

[24] O. Pavlosiuk, P. Swatek, D. Kaczorowski, and P. Wiśniewski, Phys. Rev. B 97, 235132 (2018).

[25] L.-K. Zeng, R. Lou, D.-S. Wu, Q. N. Xu, P.-J. Guo, L.-Y. Kong, Y.-G. Zhong, J.-Z. Ma, B.-B. Fu, P. Richard, P. Wang, G. T. Liu, L. Lu, Y.-B. Huang, C. Fang, S.-S. Sun, Q. Wang, L. Wang, Y.-G. Shi, H. M. Weng, H.-C. Lei, K. Liu, S.-C. Wang, T. Qian, J.-L. Luo, and H. Ding, Phys. Rev. Lett. 117, 127204 (2016).

[26] J. He, C. Zhang, N. J. Ghimire, T. Liang, C. Jia, J. Jiang, S. Tang, S. Chen, Y. He, S.-K. Mo, C. C. Hwang, M. Hashimoto, D. H. Lu, B. Moritz, T. P. Devereaux, Y. L. Chen, J. F. Mitchell, and Z.-X. Shen, Phys. Rev. Lett. 117, 267201 (2016).

[27] S. Sun, Q. Wang, P.-J. Gou, K. Liu, and H. Lei, New J. Phys. 18, 082002 (2016)

[28] O. Pavlosiuk, P. Swatek, and P. Wiśniewski, Sci. Rep. 6, 38691 (2016).

[29] T. Kasuya, M. Sera, Y. Okayama, and Y. Haga, J. Phys. Soc. Jpn. 65, 160 (1995).

[30] O. Pavlosiuk, M. Kleinert, P. Swatek, D. Kaczorowski, and P. Wiśniewski, Sci. Rep. 7, 12822 (2017).

[31] To date, only limited information on the lattice parameter [32], band-structure calculations [33], and crude estimates of the transport properties on polycrystalline samples were reported for lutetium monoarsenide [34].

[32] J. B. Taylor, L. D. Calvert, J. G. Despault, E. J. Gabe, and J. J. Murray, J. Less-Common Met. 37, 217 (1974).

[33] S. M. Said, F. B. Zid, C. M. Bertoni, and S. Ossicini, Eur. Phys. J. B 23, 191 (2001).

[34] O. Żogal, R. Wawryk, M. Matusiak, and Z. Henkie, J. Alloys Compd. 587, 190 (2014).

[35] See Supplemental Material at http://link.aps.org/supplemental/ 10.1103/PhysRevResearch.1.032016 for methods, further details on the basic properties as well as Shubnikov-de Haas oscillations and Hall-effect data. Data showing the negative longitudinal magnetoresistance are provided in Sec. VIII, while Sec. XI contains an enlarged discussion of the band structure calculation.

[36] H.-Y. Yang, T. Nummy, H. Li, S. Jaszewski, M. Abramchuk, D. S. Dessau, and F. Tafti, Phys. Rev. B 96, 235128 (2017).

[37] The XMR in LuAs does not show any tendency to saturation even up to $70 \mathrm{~T}$, as inferred from our further experiments in a pulsed-field magnet at the Hochfeld-Magnetlabor Dresden (HLD-EMFL) [S. Arsenijevic (private communication)]. 
[38] B. Fauqué, X. Yang, W. Tabis, M. Shen, Z. Zhu, C. Proust, Y. Fuseya, and K. Behnia, Phys. Rev. Mater. 2, 114201 (2018).

[39] R. Küchler, T. Bauer, M. Brando, and F. Steglich, Rev. Sci. Instrum. 83, 095102 (2012).

[40] R. W. Keyes, Solid State Phys. 20, 37 (1968).

[41] J.-P. Michenaud, J. Heremans, M. Shayegan, and C. Haumont, Phys. Rev. B 26, 2552 (1982).

[42] J. Heremans, J.-P. Michenaud, M. Shayegan, and G. Dresselhaus, J. Phys. C 14, 3541 (1981).
[43] B. S. Chandrasekhar and E. Fawcett, Adv. Phys. 20, 775 (1971).

[44] A. V. Gold and P. W. Schmor, Canad. J. Phys. 54, 2445 (1976).

[45] D. Shoenberg, Magnetic Oscillations in Metals (Cambridge University Press, Cambridge, UK, 1984).

[46] B.-B. Fu, C.-J. Yi, T.-T. Zhang, M. Caputo, J.-Z. Ma, X. Gao, B. Q. Lv, L.-Y. Kong, Y.-B. Huang, P. Richard, M. Shi, V. N. Strocov, C. Fang, H.-M. Weng, Y.-G. Shi, T. Qian, and H. Ding, Sci. Adv. 5, eaau6459 (2019). 